\title{
Variations in PET/CT Methodology for Oncologic Imaging at U.S. Academic Medical Centers: An Imaging Response Assessment Team Survey
}

\author{
Michael M. Graham ${ }^{1}$, Ramsey D. Badawi ${ }^{2}$, and Richard L. Wahl ${ }^{3}$ \\ ${ }^{1}$ University of Iowa, Iowa City, Iowa; ${ }^{2}$ University of California-Davis Medical Center, Sacramento, California; and ${ }^{3}$ Johns Hopkins \\ University School of Medicine, Baltimore, Maryland
}

\begin{abstract}
In 2005, 8 Imaging Response Assessment Teams (IRATs) were funded by the National Cancer Institute $(\mathrm{NCl})$ as supplemental grants to existing $\mathrm{NCl}$ Cancer Centers. After discussion among the IRATs regarding the need for increased standardization of clinical and research PET/CT methodology, it became apparent that data acquisition and processing approaches differ considerably among centers. To determine the variability in detail, a survey of IRAT sites and IRAT affiliates was performed. Methods: A 34-question instrument evaluating patient preparation, scanner type, performance approach, display, and analysis was developed. Fifteen institutions, including the 8 original IRATs and 7 institutions that had developed affiliate IRATs, were surveyed. Results: The major areas of variation were ${ }^{18} \mathrm{~F}-\mathrm{FDG}$ dose (259-740 MBq [7-20 mCi]) uptake time (45-90 min), sedation (never to frequently), handling of diabetic patients, imaging time (2-7 $\mathrm{min} /$ bed position), performance of diagnostic CT scans as a part of PET/CT, type of acquisition (2-dimensional vs. 3-dimensional), CT technique, duration of fasting (4 or $6 \mathrm{~h}$ ), and (varying widely) acquisition, processing, display, and PACS software-with 4 sites stating that poor-quality images appear on PACS. Conclusion: There is considerable variability in the way PET/CT scans are performed at academic institutions that are part of the IRAT network. This variability likely makes it difficult to quantitatively compare studies performed at different centers. These data suggest that additional standardization in methodology will be required so that PET/CT studies, especially those performed quantitatively, are more comparable across sites.
\end{abstract}

Key Words: oncology; PET/CT; quality assurance; IRAT; PETCT; standardization; variation

J Nucl Med 2011; 52:311-317

DOI: 10.2967/jnumed.109.074104

\section{$\mathbf{P}$} ET with ${ }^{18}$ F-FDG has been established as a broadly useful technique in cancer imaging, especially in cancer diagnosis, staging, and treatment response assessment. The utility of the PET/CT approach has been widely appreciated, and randomized trials of PET in lung cancer have

Received Dec. 19, 2009; revision accepted Jun. 29, 2010.

For correspondence or reprints contact: Michael M. Graham, Department of Radiology 3863 JPP, University of lowa, 200 Hawkins Dr., lowa City, IA 52242. E-mail: michael-graham@uiowa.edu

COPYRIGHT $\odot 2011$ by the Society of Nuclear Medicine, Inc. been shown to reduce rates of unnecessary thoracotomies (1-3). The Centers for Medicare and Medicaid Services have approved PET with ${ }^{18} \mathrm{~F}-\mathrm{FDG}$ for a broad range of indications in most cancers, supporting the value of the method. However, comparing PET studies from institution to institution can be challenging, in part because of methodologic variabilities.

In 2005, 8 Imaging Response Assessment Teams (IRATs) were funded by the National Cancer Institute (NCI) as supplemental grants to existing NCI-designated Cancer Centers. The major rationale for supporting these teams was to increase the appropriate use of quantitative medical imaging in clinical trials. In addition, through annual meetings and frequent telephone conference calls, the 8 original IRATs, as well as other additional nonfunded IRATs, have worked together on several group initiatives.

One of the IRAT national groups was the PET/CT subcommittee, chaired by 2 authors of this paper, Michael Graham and Richard Wahl. After some discussion about the need for and feasibility of standardizing both clinical and research PET/CT methodology, the IRAT group began to realize that, although the members of the group represented major academic imaging programs, data acquisition and processing differed considerably among the centers. Accordingly, the group found it appropriate to survey its members to see how they were conducting clinical PET/ $\mathrm{CT}$ - this being a needed starting point before any meaningful standardization of imaging protocols could occur.

This paper presents the results of that survey and summarizes the results.

\section{MATERIALS AND METHODS}

A series of teleconferences of the PET/CT subcommittee resulted in iterative development of a standard survey for PET/ CT for oncologic applications. Several general areas of interest were addressed, including patient preparation; methods for performance of the scan; and display, analysis, review, and archiving. The complete survey is shown in Figures 1 and 2, and the questions are summarized in Table 1. Sites that had more than 1 PET/CT system filled out the scanner-specific questions for each scanner at the institution. Ambiguous or blank answers were clarified before the data were finalized and summarized. 


\begin{tabular}{|c|c|}
\hline The IRAT Network & $\begin{array}{l}\text { 11. Low carbohydrate diet recommended one or more days before PET study? } \\
\text { Yes_ No_ }\end{array}$ \\
\hline Multi-Center PET/CT Form for Clinical Oncology & $\begin{array}{l}\text { 12. Estimated Frequency (\%) of Different Strategies used for Preparation of Diabetic } \\
\text { Patients }\end{array}$ \\
\hline 1. Name of institution: & (Total of all answers for 13 should add to $100 \%$ ): \\
\hline & 12a. No special preparation __\% \\
\hline 2. IRAT investigator(s) filling in form (physician, physicist other): & 12b. Early AM patient, overnight fasting, all diabetic medications withheld _ $\%$ \\
\hline Name: & 12c. Early AM patient, overnight fasting, some diabetic medications allowed __ $\%$ \\
\hline $\begin{array}{l}\text { e-mail: } \\
\text { _Physician _Physicist } \_ \text {Technologist }{ }^{\text {Phone }} \text { other (specify) }\end{array}$ & List: all oral meds \\
\hline Date form filled in: & 12d. Early AM patient, overnight fasting, all diabetic medications allowed _ $\%$ \\
\hline Patient Dose and Preparation & $\begin{array}{l}\text { 12e. Early PM patient, light breakfast, all diabetic medications (including insulin) } \\
\text { allowed _ \% }\end{array}$ \\
\hline 3. Administered dose of FDG (low to high) & $\begin{array}{l}\text { 12f. Early PM patient, light breakfast, some diabetic medications allowed __ } \% \\
\text { List: }\end{array}$ \\
\hline 3a. Adult: Administered dose of FDG (low to high): & \\
\hline _to __ $\mathrm{mCi}$ or __to & 12g. Blood glucose level titrated with IV insulin __\% \\
\hline 3b. Children: $\overline{\text { Administered dose of FDG (low to high): }}$ & 12h. Other_\% $\%$ \\
\hline Comment: ${ }^{\text {to }}{ }^{\mathrm{mCi} \text { or }}{ }^{\text {to }}{ }^{\mathrm{mCi} / \mathrm{Kg}}$ & Describe: \\
\hline & 13. Blood glucose measured before FDG injection? (Check all that apply.) \\
\hline 4. Uptake period: __ to _ minutes & 13a. No __ 13b. Yes, all patients __ 13c. Yes, diabetic patients only ___ \\
\hline Comment: & $\begin{array}{l}\text { 13d. Yes, other criterion } \\
\text { If yes, Describe: }\end{array}$ \\
\hline 5. CT contrast used during transmission scan: & \\
\hline 5a. None_ ${ }_{-} \%$ 5b. Oral positive _ $\%$ 5c. Oral negative _ $\%$ 5d. Intravenous & $\begin{array}{l}\text { 14. Blood glucose cut-off: } \\
\text { Comment: }\end{array}$ \\
\hline $\begin{array}{l}\text { 6. What fraction of patients have a separate diagnostic } \mathrm{CT} \text { acquisition: } \\
\text { 6a. With contrast _ } \% 6 \text { b. Without contrast _ } \%\end{array}$ & $\frac{\text { Scanner, Acquisition, and Reconstruction Parameters }}{\text { (Please complete for each type of scanner) }}$ \\
\hline $\begin{array}{l}\text { 7. How often is bladder catheterization performed?__ } \% \\
\text { Comment: }\end{array}$ & $\begin{array}{l}\text { 15. Scanner number ___ of ___ scanners for this institution. } \\
\text { (i.e., number } 1 \text { of } 2 \text { ) }\end{array}$ \\
\hline $\begin{array}{l}\text { 8. How often is sedation used? _ } \% \\
\text { Comment: }\end{array}$ & 16. PET scanner manufacturer and model: \\
\hline 9. Percent of cases with IV contrast: & 17. PET scanner software version number: \\
\hline a. Head and neck __ $\%$ b. Lung __ $\%$ c. Lymphoma __ $\%$ & 18. Emission scan acquisition mode (percent): \\
\hline d. Colon__\% e. Other_\% & 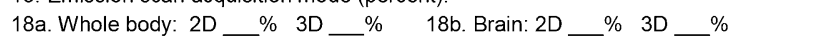 \\
\hline
\end{tabular}

FIGURE 1. The complete survey: page 1.

The 34-question survey was completed by the following 15 sites: the University of Iowa, Johns Hopkins University, Ohio State University, the University of Pittsburgh, Roswell Park Cancer Institute, Washington University, the University of Washington, the University of Wisconsin, Memorial Sloan-Kettering Cancer Center, the University of Arizona, the Dana-Farber Cancer Institute, the University of Colorado, Georgetown University, Vanderbilt University, and the University of California-Davis.

\section{RESULTS}

\section{Patient Dose and Preparation}

The average administered ${ }^{18} \mathrm{~F}$-FDG dose for adults varies from 259 to $740 \mathrm{MBq}(7-20 \mathrm{mCi})$. At least 2 institutions give as much as $925 \mathrm{MBq}(25 \mathrm{mCi})$. For those sites (only 3) that reported dose per kilogram, the range is 5.18-8.14 $\mathrm{MBq}(0.14-0.22 \mathrm{mCi}) / \mathrm{kg}$. Pediatric dose modifications were not surveyed.

The uptake time after injection varies from 45 to $90 \mathrm{~min}$. Most try for $60 \mathrm{~min}$ but tolerate deviations from 45 to $90 \mathrm{~min}$. Five sites image as late as $90 \mathrm{~min}$ occasionally or usually, and 4 sites image as early as $45 \mathrm{~min}$. For brain imaging, at least 1 site images as early as $30 \mathrm{~min}$.
Most sites do the CT transmission scan before injection of CT intravenous contrast material.

Most sites do diagnostic CT 15\%-50\% of the time, 4 sites never do diagnostic CT, and 1 site does diagnostic CT in all patients. Almost all sites use intravenous contrast material for diagnostic CT.

Bladder catheterization is rarely or never used at any of the sites.

Ten sites almost never use sedation ( $<3 \%$ of the time). Others use it more frequently, particularly in head and neck cancer.

The percentage of cases in which intravenous contrast material is used varies widely by disease type. The most common use is in head and neck cancer (average, 28\%; range, $0 \%-100 \%)$, followed by lymphoma (18\%, 0\%-95\%), lung cancer $(13 \%, 0 \%-80 \%)$, and colon cancer $(13 \%, 0 \%-95 \%)$.

The recommended minimum duration for fasting was evenly split between 4 and $6 \mathrm{~h}$, although 1 site suggested $12 \mathrm{~h}$.

The sites were evenly split on recommending a lowcarbohydrate diet on the day before the PET study.

A wide range of different strategies is used for preparation of diabetic patients: performing PET/CT early in the morning 


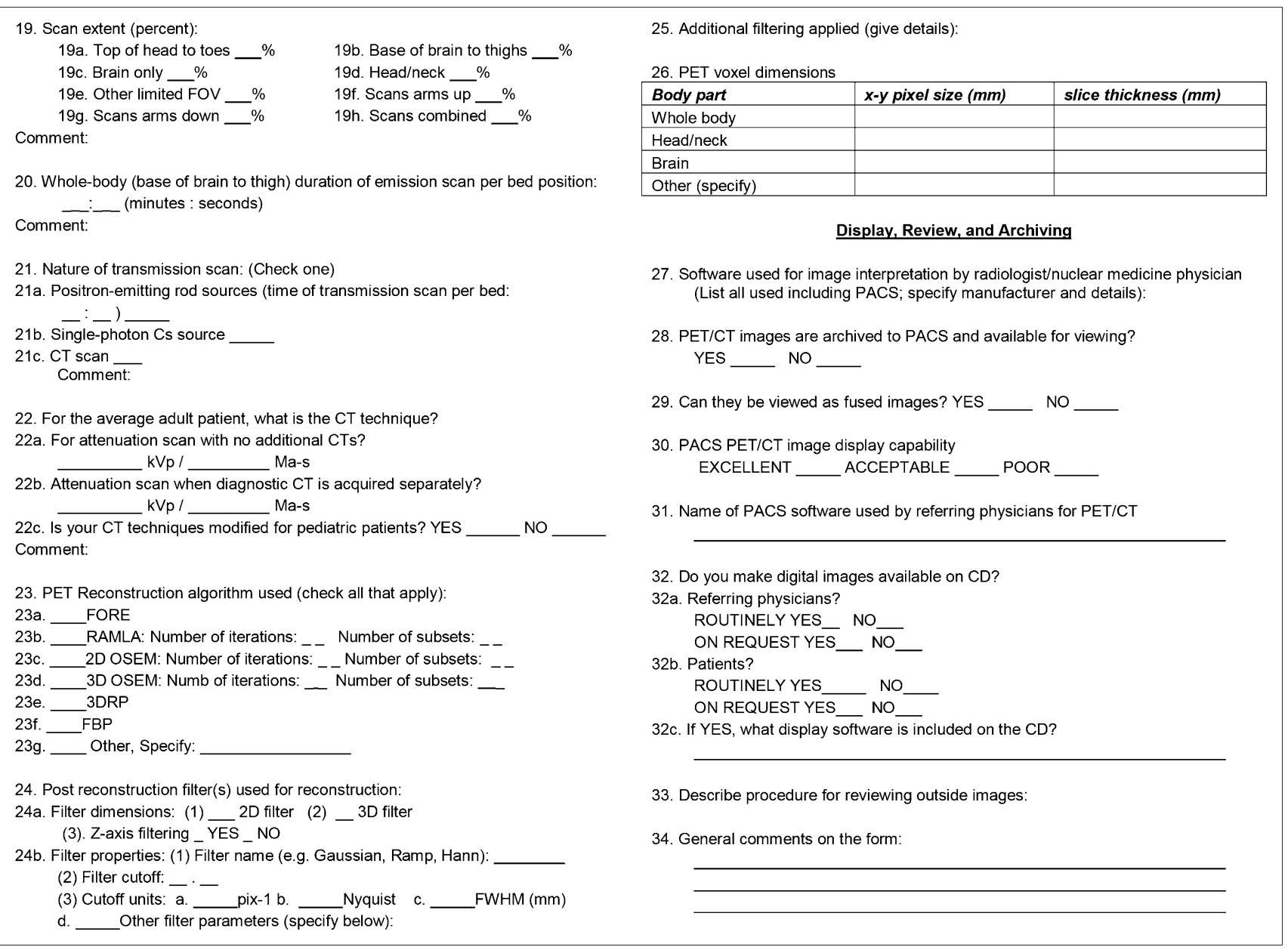

FIGURE 2. The complete survey: page 2.

after an overnight fast and holding all diabetic medications (applied in $40 \%$ of diabetic studies); performing PET/CT early in the morning after an overnight fast and holding some diabetic medications (applied in 27\%); performing PET/CT early in the morning after an overnight fast and allowing all diabetic medications (applied in 7\%); allowing an earlymorning light breakfast and all diabetic medications (applied in 15\%); allowing an early-morning light breakfast and some diabetic medications (applied in 11\%); and titrating with intravenous insulin when necessary (almost never applied).

All but one of the sites measure blood glucose levels before the ${ }^{18} \mathrm{~F}-\mathrm{FDG}$ injection. The single exception is a site that measures blood glucose levels only in diabetic or research patients. Most sites have a policy of not performing a study on a patient whose blood glucose level is above $200 \mathrm{mg} / \mathrm{dL}$. One site has a cutoff of $180 \mathrm{mg} / \mathrm{dL}$, another has a cutoff of $220 \mathrm{mg} / \mathrm{dL}$, and one does not have a cutoff. Two indicated they have a lower limit of $70 \mathrm{mg} / \mathrm{dL}$.

\section{Scanner, Acquisition, and Reconstruction Parameters}

Eight sites have GE Healthcare scanners, 5 have Siemens Healthcare scanners, and 2 have both GE and Siemens scanners.
Apparently, the different sites all have different versions of PET scanner software, although this apparent difference may be partly due to lack of rigor in determining the version numbers. Clearly, substantial variability is present-even within the same manufacturer and scanner model.

The emission acquisition for whole-body imaging is 3-dimensional (3D) for 8 systems (all Siemens) and 2-dimensional (2D) for 13 systems (all GE). Brain imaging, when reported separately, is almost always 3D (7 sites), although 2 sites report doing 2D brain studies.

Most PET/CT studies are done from the base of the brain to the thighs $(83 \%)$. Other scan extents include top of head to toes (7\%), brain only (5\%), and head and neck only $(5 \%)$. Approximately $60 \%$ of studies are done with the arms up and $40 \%$ with the arms down. Arm position was almost certainly related to the type of examination-that is, neck versus chest or body.

The duration of the emission acquisition per bed position for whole-body scans ranges from 2 to $7 \mathrm{~min}$. The sites with longer scans generally administer lower doses of ${ }^{18} \mathrm{~F}-\mathrm{FDG}$.

The transmission scans for all PET/CT scanners are done using the CT portion of the PET/CT scanner. 


\begin{tabular}{|c|c|}
\hline Category & Question \\
\hline Patient dose and preparation & $\begin{array}{l}\text { 1. Administered dose of }{ }^{18} \mathrm{~F}-\mathrm{FDG}: \\
\text { 2. Uptake period in minutes: } \\
\text { 3. Is CT contrast used during the transmission scan? } \\
\text { 4. Fraction of patients who have a separate diagnostic CT acquisition: } \\
\text { 5. How often bladder catheterization is performed: } \\
\text { 6. How often sedation is used: } \\
\text { 7. Percentage of cases with intravenous contrast: } \\
\text { 8. Minimum duration of fasting before }{ }^{18} \mathrm{~F}-\mathrm{FDG} \text { injection: } \\
\text { 9. Is a low-carbohydrate diet recommended one or more days before PET study? } \\
\text { 10. Estimated frequency of different strategies used for preparation of diabetic } \\
\quad \text { patients: } \\
\text { 11. Is the blood glucose measured before }{ }^{18} \mathrm{~F}-\mathrm{FDG} \text { injection? } \\
\text { 12. Blood glucose cutoff: }\end{array}$ \\
\hline $\begin{array}{l}\text { Scanner, acquisition, and reconstruction } \\
\text { parameters }\end{array}$ & $\begin{array}{l}\text { 13. PET scanner manufacturer and model: } \\
\text { 14. PET scanner software version number: } \\
\text { 15. Emission scan acquisition mode ( } 2 \mathrm{D} \text { vs. } 3 \mathrm{D} \text { for whole body and brain): } \\
\text { 16. Scan extent (top of head to toes, base of brain to thighs, arms up, arms down, } \\
\quad \text { etc.): } \\
\text { 17. For whole-body scan, duration of emission scan per bed position: } \\
\left.\text { 18. Nature of transmission scan (CT, }{ }^{68} \mathrm{Ge} \text { rod source, }{ }^{133} \mathrm{Cs}\right) \text { : }\end{array}$ \\
\hline Display, review, and archiving & $\begin{array}{l}\text { 19. CT technique used for the average adult patient: } \\
\text { 20. PET reconstruction algorithm used: } \\
\text { 21. Postreconstruction filter(s) used for reconstruction: } \\
\text { 22. PET voxel dimensions: } \\
\text { 23. Software used for image interpretation by radiologist/nuclear medicine physician: } \\
\text { 24. Are PET/CT images archived to PACS and available for viewing? } \\
\text { 25. Can PET/CT images on PACs be viewed as fused images? } \\
\text { 26. Quality of PACS PET/CT image display capability: } \\
\text { 27. Name of PACS software used by referring physicians for PET/CT: } \\
\text { 28. Do you make digital images available on compact disk for referring physicians } \\
\quad \text { or patients? } \\
\text { 29. Type of display software included on the compact disks you provide: } \\
\text { 30. Procedure for reviewing outside images: }\end{array}$ \\
\hline
\end{tabular}

For the average adult patient, the $\mathrm{CT}$ technique varies moderately. Most studies are done at $120 \mathrm{kVp}$ (10 CT systems), although 2 other energies are also used: $140 \mathrm{kVp}$ (4) and $160 \mathrm{kVp}(1)$. At least 4 sites adjust amperage automatically, presumably using appropriate software incorporated into their systems. Of the sites with a fixed amperage, most use $50 \mathrm{mAs}$ (4), although the range is 8-120 mAs. Most sites (13) adjust CT dose for pediatric patients, although 2 do not.

The most common PET reconstruction algorithm used is 2D ordered-subsets expectation maximization (OSEM) with 2 iterations and 20-30 subsets (total of 12 scanners). Fourier rebinning 2D OSEM is used by 4 scanners. 3D OSEM is used by 3 scanners. Filtered backprojection is not used by any of the sites.

2D postreconstruction filtering is used as part of image reconstruction for 14 scanners. 3D filtering is used for 4 scanners. $z$-axis filtering is used at least for some of the images for 13 scanners.

The most common PET voxel dimensions (in $\mathrm{mm}$ ) for GE scanners (8/13) are 4.69 for $x, 4.69$ for $y$, and 3.27 for $z$. For Siemens scanners (5/9) the dimensions are 4.06 for $x$,
4.06 for $y$, and 3.37 for $z$. Generally, smaller voxels are used for dedicated brain imaging.

\section{Display, Review, and Archiving}

Sites with GE scanners usually use GE Xeleris software for image interpretation by radiologists and nuclear medicine physicians. Half the sites with Siemens scanners use Siemens software (Esoft or Leonardo). The other sites use software by MedImage, MIM Software Inc., or Phillips (Stentor, now iSite).

All sites archive their PET/CT images to a PACS, where the images are available for viewing. At 12 sites, fused images can be viewed on the PACS, although at some sites image fusion is achieved only through saving screenshots.

The display quality of PACS PET/CT images varies markedly across the sites: 5 sites assess the quality of the PACS image display as excellent, 6 as acceptable, and 4 as poor. A wide range of PACS software is used for the referring physicians. The software packages include those by McKesson Corp. (3 sites), Philips (iSite) (5 sites), Agfa Healthcare (2 sites), UltraVisual Medical Systems (1 site), and Emageon Inc. (1 site). 
Most sites make digital images available on compact disk for referring physicians and patients. A wide range of viewing software is supplied with the compact disks. The most common are eFilm lite (Merge Healthcare Inc.) (4) and MIMviewer (MIM Software Inc.) (3). Other sites used packages by Hermes Medical Solutions, Philips (iSite), MedImage, and GE Healthcare (Centricity).

Most sites load outside DICOM images onto their PACS or PET image viewing systems for review.

\section{DISCUSSION}

Surprisingly little information has been available on the variability in PET practices in the United States. This survey showed that there are many commonalities but also that, across various institutions with considerable expertise in PET, there is remarkably wide variability in some aspects of how clinical PET studies are performed. It is likely that this large variation arises because of the lack of a rigorously proven and validated optimum method for conducting the studies. In the absence of a standard, each site has chosen what seems to be the most reasonable approach.

The wide variation certainly means that it is quite difficult to use the retrospective data of a site in any meaningful multicenter quantitative analysis of efficacy, especially if absolute quantitation is required across centers. This wide variation also impedes the ability of the various sites to participate in prospective clinical trials, because baseline studies are often done before a patient is recruited into a trial. If the standard technique varies markedly from the required technique in the study, the baseline scan will often have to be repeated.

Another significant problem with this wide variation is that sensitivities and specificities for a specific clinical settingfor example, staging lung cancer-potentially could be different at each institution. Quantitative thresholds, such as SUV, will also differ. This is a reason that health technology assessment experts often regard the literature on ${ }^{18} \mathrm{~F}-\mathrm{FDG}$ PET/CT as limited in adequately justifying the utility of the studies.

Several guidelines on oncologic imaging with ${ }^{18} \mathrm{~F}-\mathrm{FDG}$ PET/CT have been developed by the NCI (4), the Netherlands and European groups $(5,6)$, and the American College of Radiology Imaging Network (ACRIN) (7). Similar efforts are under way by other groups, including the Quantitative Imaging Biomarkers Alliance and the Uniform Protocols for Imaging in Clinical Trials group. Table 2 shows how the recommendations of the NCI, the European group, and ACRIN compare with the findings from the IRAT survey for several areas of concern.

The following parameters have moderate variation among the sites surveyed but are reasonably close to the NCI, European, and ACRIN recommendations. It is likely that broad agreement can be achieved relatively easily regarding these parameters.

\section{Administered Dose}

Generally, the recommendations of the European group are lower than those in the United States. It appears that
$370-555 \mathrm{mBq}(10-15 \mathrm{mCi})$ is reasonable, although with newer tomographs and with the increased general concern about patient radiation exposure (8), recommendation of a lower range may be appropriate. In general, it would be more appropriate to specify an administered dose per kilogram of body weight, because patient weight ranges widely.

\section{Uptake Time}

The general recommendation is that imaging take place $60 \pm 10 \mathrm{~min}$ after injection of the ${ }^{18} \mathrm{~F}-\mathrm{FDG}$. This is the only parameter that is essentially empiric and convenient, but the value is also probably not optimal. Numerous papers show that tumor uptake continues to rise over time and that tumor-to-background ratio improves over time. The problem with defining 60 min now, for practical reasons, as standard for PET/CT studies is that this timing may become firmly established despite multiple existing and future studies that show the utility of imaging later. This issue should be carefully considered before $60 \mathrm{~min}$ is defined as standard.

\section{Duration of Fasting}

It appears that the most reasonable recommendation will be that fasting should be for at least $4 \mathrm{~h}$ and preferably for at least $6 \mathrm{~h}$.

\section{Recommendation for Low-Carbohydrate Diet}

The NCI and half the IRAT sites recommend a lowcarbohydrate diet to their patients. It is not really known how well patients adhere to the recommendation or how effective it is in improving the quality or reproducibility of ${ }^{18}$ F-FDG PET/CT studies. In the absence of any compelling evidence, this recommendation is difficult to support, and patient compliance is difficult to verify.

\section{Blood Glucose Cutoff}

There seems to be broad agreement that oncologic ${ }^{18} \mathrm{~F}$ FDG PET/CT studies should certainly not be done if the blood glucose level is above $200 \mathrm{mg} / \mathrm{dL}$ (11 mmol); however, both the NCI and the European group suggest that the limit should be much lower: $120 \mathrm{mg} / \mathrm{dL}$. This suggestion likely reflects concerns about standardization in clinical trials, whereas the cutoff of $200 \mathrm{mg} / \mathrm{dL}$ at the surveyed sites reflects the practical operation in a clinical PET center.

\section{Emission Scan Duration per Bed Position}

The appropriate duration of imaging per bed position depends on the type of tomograph (2D vs. 3D), the amount of overlap per bed position, the administered dose, and body weight. Recommending an optimal time or range of times seems inappropriate. Rather, imaging time should be based on a calculation involving administered dose and body weight, or on the true counting rate recorded from the patient.

\section{CT Technique}

A wide variation, particularly in amperage, was seen at the IRAT sites. The European recommendations are somewhat lower than the recommendations of most IRAT sites. Because of increasing concern about radiation exposure (8), 
TABLE 2

Comparison of IRAT Survey Results with Recommendations of NCl, European Group, and ACRIN

\begin{tabular}{|c|c|c|c|c|}
\hline Parameter & $\mathrm{NCl}$ & European group & ACRIN & IRAT survey \\
\hline Administered dose & $\begin{array}{r}370-740 \mathrm{MBq} \\
(10-20 \mathrm{mCi})\end{array}$ & $\begin{array}{l}5 \mathrm{mBq} / \mathrm{kg} \text { for } 2 \mathrm{D}, 2.5 \\
\mathrm{MBq} \text { for } 3 \mathrm{D} \text { (370 } \mathrm{MBq} \\
\text { [10 } \mathrm{mCi} \text { for } 2 \mathrm{D}, 185 \\
\mathrm{MBq}[5 \mathrm{mCi}] \text { for } 3 \mathrm{D} \text { for } \\
70-\mathrm{kg} \text { patient) }\end{array}$ & $\begin{array}{l}\text { Dose is specific } \\
\text { to each trial }\end{array}$ & $\begin{array}{l}259-740 \mathrm{MBq} \\
\quad(7-20 \mathrm{mCi})\end{array}$ \\
\hline Uptake time & $50-70 \mathrm{~min}$ & $55-65 \mathrm{~min}$ & $50-70 \mathrm{~min}$ & 45-90 $\min$ \\
\hline $\begin{array}{l}\text { Duration of } \\
\text { fasting }\end{array}$ & Minimum of $4 \mathrm{~h}$ & $4 \mathrm{~h}$, prefer 6 & Minimum of $4 \mathrm{~h}$ & 4 or 6 h (evenly split) \\
\hline $\begin{array}{l}\text { Recommendation } \\
\text { for low- } \\
\text { carbohydrate diet }\end{array}$ & $\begin{array}{l}\text { Recommend for } \\
24 \mathrm{~h} \text { before }\end{array}$ & Not mentioned & $\begin{array}{l}\text { Recommend for } 24 \mathrm{~h} \\
\text { before }\end{array}$ & $\begin{array}{l}\text { Half the sites } \\
\text { recommend }\end{array}$ \\
\hline $\begin{array}{l}\text { Blood glucose } \\
\text { cutoff }\end{array}$ & $\begin{array}{l}<120 \mathrm{mg} / \mathrm{dL} \text { for } \\
\text { nondiabetic } \\
\text { patients; } 150-200 \\
\text { mg/dL for diabetic } \\
\text { patients }\end{array}$ & $\begin{array}{l}\text { Reschedule if more } \\
\text { than } 126 \mathrm{mg} / \mathrm{dL}\end{array}$ & $\begin{array}{l}\text { Reschedule if more than } \\
150-200 \mathrm{mg} / \mathrm{dL}\end{array}$ & $\begin{array}{l}\text { Reschedule if more than } \\
200 \mathrm{mg} / \mathrm{dL}\end{array}$ \\
\hline $\begin{array}{l}\text { Duration of } \\
\text { emission scan per } \\
\text { bed position }\end{array}$ & Not mentioned & $\begin{array}{l}\text { Typically } 5 \text { min } \\
\text { per bed position }\end{array}$ & $\begin{array}{l}\text { Use manufacturer } \\
\text { recommendations }\end{array}$ & $2-7 \min$ \\
\hline CT technique & Not mentioned & $30 \mathrm{mAs}$ or less & $\begin{array}{l}\text { Technique is specific to } \\
\text { each trial }\end{array}$ & $\begin{array}{l}\text { Variable and } \\
\text { center-specific }\end{array}$ \\
\hline $\begin{array}{l}\text { Reconstruction } \\
\text { algorithms }\end{array}$ & $\begin{array}{l}\text { No specific } \\
\text { recommendations }\end{array}$ & $\begin{array}{l}\text { Specific for GE, } \\
\text { Siemens, and Phillips }\end{array}$ & $\begin{array}{l}\text { Use manufacturer } \\
\text { recommendations }\end{array}$ & $\begin{array}{l}\text { 2D OSEM, } 2 \text { iterations. } \\
\text { 20-30 subsets }\end{array}$ \\
\hline $\begin{array}{l}\text { How to handle } \\
\text { diabetic } \\
\text { patients }\end{array}$ & $\begin{array}{l}\text { Scan in morning } \\
\text { after overnight fast } \\
\text { and before first use } \\
\text { of medication }\end{array}$ & $\begin{array}{l}\text { Specific } \\
\text { recommendations for } \\
\text { type I and type } ~{ }^{*}\end{array}$ & $\begin{array}{l}\text { Scheduled in morning } \\
\text { with instructions } \\
\text { provided in consultation } \\
\text { with primary physician }\end{array}$ & $\begin{array}{l}\text { Wide variation, } \\
\text { as described in text }\end{array}$ \\
\hline
\end{tabular}

*Type I: Study will be scheduled preferably at end of morning; patient will have normal breakfast at 7:00 AM and use regular dose of insulin, followed by fasting for at least $4 \mathrm{~h}$. Type II: Study will be scheduled preferably at end of morning; patient needs to have fasted for at least $4 \mathrm{~h}$; intake of water is recommended; oral antidiabetic drugs should be continued.

it may be appropriate to recommend $30-50 \mathrm{mAs}$ or less for attenuation imaging for an average-size patient and to adjust for body size at a reasonably high pitch. Adjustment for body size, collimation, and pitch is particularly important in children (9).

Some parameters are more difficult and have wide variation at the sites surveyed and in the NCI, European, and ACRIN recommendations. These parameters, which include diabetic patients, reconstruction algorithms, voxel sizes, and analysis software, will require further investigation and discussion.

The survey showed a wide variation in how to manage diabetic subjects, and the recommendations of the NCI, European group, and ACRIN do not converge. This divergence likely reflects lack of knowledge of an optimum strategy and the fact that the final results may not be sensitive to the exact strategy. We need to pick a strategy and adopt it widely.

Reconstruction algorithms and voxel size are dependent on the options available for the various available PET/CT systems. Standardization of these parameters is essential and will require collaboration between representatives of both industry and academia. The challenge will be to ensure that the images of all major manufacturers are quantitatively comparable.

The same problem exists for display and analysis software, but the problem may be more challenging in this area because of the wider range of available software. Validation methodology is essential to ensure that all software produces the same result.

One area not included in the survey was the frequency and type of calibration at the facilities surveyed. Frequent, careful calibration is important in maintaining the accuracy and capabilities of a PET/CT system. As recommendations for quality assurance in PET/CT expand, an important recommendation to include will be calibration with a common phantom across all the sites in a clinical trial. Such calibration is also appropriate for high-quality clinical imaging and is likely to become a future standard as part of the accreditation of all systems.

The quality of the display capabilities of the PACS systems at the surveyed sites varied widely, from poor to excellent. Because high-quality display capability, including viewing fused images, definitely exists, this variability in display quality likely reflects the fact that some sites 
have upgraded their PACS system more recently than other sites.

${ }^{18}$ F-FDG PET/CT has proven to be a remarkably effective clinical tool despite the wide variation in technique used at imaging centers throughout the United States and the world. It is virtually certain that the variation in the nonsurveyed centers is considerably wider than that found in the surveyed group of academic centers. Although ${ }^{18} \mathrm{~F}$ FDG PET/CT is robust and can be used with wide variation, the technique would significantly benefit from standardization, particularly as we try to identify new indications for reimbursement, for clinical trials, and for reproducible quantitative imaging to assess response to therapy. Approaches for standardizing PET have been proposed in an NCI consensus paper (4) and, more recently, from experience in Europe $(5,6)$. Such studies suggest PET can be made more quantitatively comparable across sites by standardization of imaging approaches.

We have made some suggestions in this paper on the specific parameters that need to be standardized. This paper is not intended to be a definitive statement but points the way toward a consensus paper, which is definitely needed.

\section{CONCLUSION}

A survey applied mainly to academic PET centers participating in the IRAT network showed considerable variability in patient preparation, ${ }^{18} \mathrm{~F}-\mathrm{FDG}$ dose, CT technique, tracer uptake, imaging time, reconstruction methods, and suitability of PACS for PET/CT display. The existence of this variance despite professional guidelines suggests that results from quantitative PET analyses are likely to differ widely across centers. These data indicate that additional standardization is needed to bring about results — particularly quantitative results - that are more comparable among sites.

\section{ACKNOWLEDGMENTS}

The study was supported by IRAT supplements to NCI Cancer Center support grants at Johns Hopkins University (3P30CA006973), Memorial Sloan-Kettering Cancer Center (3P30CA008748), Ohio State University (3P30CA016058), the University of Arizona (2P30CA023074), the University of California-Davis (3P30CA093373), the University of Iowa (5P30CA086862), the University of Pittsburgh (3P30CA047904), and Washington University (5P30CA091842).

\section{REFERENCES}

1. Maziak DE, Darling GE, Inculet RI, et al. Positron emission tomography in staging early lung cancer: a randomized trial. Ann Intern Med. 2009;151:221228

2. Fischer B, Lassen U, Mortensen J, et al. Preoperative staging of lung cancer with combined PET-CT. N Engl J Med. 2009;361:32-39.

3. van Tinteren H, Hoekstra OS, Smit EF, et al. Effectiveness of positron emission tomography in the preoperative assessment of patients with suspected non-smallcell lung cancer: the PLUS multicentre randomised trial. Lancet. 2002;359:13881393

4. Shankar LK, Hoffman JM, Bacharach S, et al. Consensus recommendations for the use of ${ }^{18}$ F-FDG PET as an indicator of therapeutic response in patients in National Cancer Institute Trials. J Nucl Med. 2006;47:1059-1066.

5. Boellaard R, Oyen WJ, Hoekstra CJ, et al. The Netherlands protocol for standardisation and quantification of FDG whole body PET studies in multi-centre trials. Eur J Nucl Med Mol Imaging. 2008;35:2320-2333.

6. Boellaard R, O'Doherty MJ, Weber WA, et al. FDG PET and PET/CT: EANM procedure guidelines for tumour PET imaging: version 1.0. Eur J Nucl Med Mol Imaging. 2010;37:181-200.

7. ACRIN PET Core Laboratory Standard Operating Procedures. American College of Radiology Imaging Network Web site. Available at: www.acrin.org/ CORELABS/PETCORELABORATORY/PETSOPS/tabid/484/Default.aspx. Accessed September 3, 2010.

8. Brenner DJ, Hall EJ. Computed tomography: an increasing source of radiation exposure. N Engl J Med. 2007;357:2277-2284.

9. Alessio AM, Kinahan PE, Manchanda V, Ghioni V, Aldape L, Parisi MT. Weightbased, low-dose pediatric whole-body PET/CT protocols. J Nucl Med. 2009;50: 1570-1577. 